Nig. J. Biotech. Vol. 37(2): 126-133 (Dec. 2020)

ISSN: 01891731

Available online at

http://www.ajol.info/index.php/njb/index

and www.biotechsocietynigeria.org

DOI: https://dx.doi.org/10.4314/njb.v37i2.12

\title{
Effects of Momordica Charantia Silver Nanoparticles on the expressions of Genes Associated With Lipid Metabolism and Nephrotoxicity in Streptozotocin-Induced Rats
}

\author{
Elekofehinti 0.0. ${ }^{* 1}$ and Akinjiyan M.O' \\ ${ }^{1}$ Bioinformatics and Molecular Biology Unit, Department of Biochemistry, Federal University of \\ Technology, Akure, Ondo State, PMB 704, Nigeria
}

\begin{abstract}
Hyperlipidemia and hyperglycemia have been implicated in diabetes mellitus (DM) leading to complications such as nephropathy. Medicinal plants like Mormodica charantia (MC) have been used in the treatment of DM over the years but little is known about their mechanisms of action. This study used biotechnology tools to investigate and compare the effects of M. charantia silver nanoparticles (MCSNPs) with M. charantia extract on expressions of genes linked with nephrotoxicity, lipid and glucose metabolisms using reverse-transcriptase polymerase chain reaction (RT-PCR) in streptozotocin-induced diabetic rats. The genes investigated include kidney injury molecule-1 (KIM-1), 3hydroxyl, 3-methyl glutaryl_coA reductase (HMG-CoA reductase), peroxisome proliferatoractivated receptor alpha and gamma (PPARa and PPARY). Synthesis of MCSNPs was done using $1 \mathrm{mM}$ concentration of aqueous silver nitrate solution at ratio 1:9 (v/v). Experimental rats were induced intraperitoneally with streptozotocin $(65 \mathrm{mg} / \mathrm{kg}$ ) and divided into six groups viz: diabetic control; normal control; silver nitrate $(10 \mathrm{mg} / \mathrm{kg}) ;$ MCSNPs (50 $\mathrm{mg} / \mathrm{kg})$; Metformin $(100 \mathrm{mg} / \mathrm{kg})$ and $M$. charantia fraction $(100 \mathrm{mg} / \mathrm{kg})$. Sacrifice was done after 12 days of treatment and RT-PCR was then used to investigate gene expressions in liver and kidney tissues of the rats. The expression of HMG-CoA reductase gene was significantly upregulated $(p<0.05)$ upon treatment with $50 \mathrm{mg} / \mathrm{kg}$ MCSNPs relative to the diabetic untreated group. $M$. charantia extracts and MCSNPs significantly upregulate $(p<0.05)$ the expressions of PPAR-a and PPAR-y compared to the diabetic control. Also, a significant $(p<0.05)$ down-regulation of KIM-1 mRNA expression was observed in MCSNPs- treated group, relative to the diabetes untreated group. $M$. charantia silver nanoparticles could be a potent antidiabetic agent due to its potential to modulate genes associated with lipid metabolism and nephrotoxicity.

Keywords: Medicinal plant; Diabetes Mellitus; Silver Nanoparticles; nephrotoxicity; gene expression

*Corresponding author's email: ooelekofehinti@futa.edu.ng; solaeleko90@gmail.com

Phone number: +2348034450611
\end{abstract}

\section{Introduction}

Diabetes mellitus (DM) describes a group of metabolic disorders which is characterized by high blood glucose (blood sugar), either due to inadequate insulin secretion or action or both (WHO 2006, Elekofehinti et al., 2016; 2018). As of 2015, an estimated 415 million people were diagnosed with diabetes mellitus worldwide, this is projected to rise to 430 million in 2030 with type 2 DM making up about $90 \%$ of the cases (Shaw et al., 2010). In DM cases, there is impediment in glucose process in its catabolism as immediate energy source or anabolism as glycogen for storage, as a result of insulin deficiency, inadequacy or malfunctioning. Complications of DM can be short- term or long- term; they include nephropathy, urinary infections, retinopathy, neuropathy, cardiovascular diseases, 
ketoacidosis and weight loss (Bloomgarden, 2003; Kitabchi et al., 2009;). DM may result in kidney stone formation, partial or total kidney failure. This disorder is the major cause of kidney failure in the developed world and accounts for approximately 35 to $40 \%$ of cases of End Stage Renal Disease (ESRD) each year (Mbanya and Sobngwi, 2003).

Conventional antidiabetic drugs like metformin, acarbose, sulphonylureas and biguanides among others have been found to have side effects, unlike the use of medicinal plants which have been reported to have little or no side effects (Omonkhua et al., 2012; Elekofehinti et al., 2014; Ahn, 2017). Phytomedicine or Plant-based medicine has been used cost-effectively to treat diabetes mellitus worldwide. In many parts of the planet, especially poor countries, this may be the only form of therapy available to treat diabetic patients. There are several documented evidences about anti-diabetic herbal plants like Mormodica charantia (Patel et al., 2010). Hypoglycemic herbs increase insulin secretion, enhance glucose uptake by adipose or muscle tissues and inhibit glucose absorption from intestine and glucose production from liver (Hui et al., 2009).

Nanoparticles can be used as carriers of drugs in order to overcome the enzymatic degradation in the stomach and improve permeation through the gastrointestinal (GI) tract. They can be synthesized by solid reaction, co-precipitation, chemical reaction, sol gel method, microwave irradiation and green synthesis among others (Shameli et al., 2010). The various nanoparticles include gold, silver, copper, iron, palladium; and among these, silver nanoparticles are known to be excellent (Zhang et al., 2016).

One of the major concerns in drug delivery is bioavailability at the target sites and conventional drugs/herbs administered through oral route may not get to the target tissues at the required dose. This study was designed to synthesize silver nanoparticles using a lower dose of $M$. charantia, and compare its effects with a higher dose of $M$. charantia aqueous extract on selected genes associated with nephrotoxicity, lipid and glucose metabolism in streptozotocin-induced diabetic rats. The genes investigated in the study include kidney injury molecule-1 (KIM-1), 3-hydroxyl, 3-methyl glutaryl coA reductase (HMG-CoA reductase), peroxisome proliferator-activated receptor alpha and gamma (PPARa and PPARY).

\section{Materials and Methods \\ Materials}

Chemicals, reagents and solvents used during this research were of analytical status. Silver nitrate and methanol were products of Sigma Chemical Co. (USA). Trizol, reverse transcriptase, gel loading dye, TBE buffer and DNA ladder were purchased from Inqaba Biotec South Africa

\section{Methods}

Bitter melon leaves (Momordica charantia) were collected from within Akure, Ondo State, Nigeria. They were air- dried and grou nd . The bitter lemon nanoparticles were synthesized according to the modified method of Bharathi et al. (2017). Briefly, filtrate from the plant extract was added to $1 \mathrm{mM}$ concentration of aqueous silver nitrate solution at the ratio 1: $9(\mathrm{v} / \mathrm{v})$. The resulting solution was then left for about 24hrs for the nanoparticles to be fully formed. Change in colour of the solution from dark green to light brown was observed, which became deeper after $24 \mathrm{hrs}$, indicating the formation of silver nanoparticles. The bio reduction of $\mathrm{Ag}^{+}$ ions to $\mathrm{Ag}^{0}$ was monitored using a UV-Vis spectrophotometer to take the absorbance, since nanoparticles formation is evidenced by the appearance of surface plasmon resonance band at $420-470 \mathrm{~nm}$. The nanoparticles solution was then freeze- dried and stored at room temperature to enhance stability (Abdelwahed et al., 2006). The nanoparticles were further characterized using a Scanning Electron Microscope (SEM) and Fourier Transformed Infrared Spectroscopy (FTIR) (Bharathi et al., 2017).

\section{Experimental design and Animal Treatment}

Male Wistar rats were used for this study. The animals were housed at the animal house of the Biochemistry Department, Federal University of Technology, Akure, Ondo State, Nigeria. The animals were then acclimatized and randomly allocated into 6 groups containing at least 6 animals per group. Diabetes mellitus was induced by intraperitoneal (i.p) injection freshly prepared and buffered ( $0.1 \mathrm{M}$ citrate, $\mathrm{pH} 4.5)$ solution of streptozotocin (STZ) at 65 $\mathrm{mg} / \mathrm{kg} /$ body weight into the overnight fasted rats. After $72 \mathrm{hrs}$, the animals with blood glucose $\geq 200 \mathrm{mg} / \mathrm{dl}$ were selected and used for subsequent experiments (Akbarzadeh et al., 2007; Elekofehinti et al., 2020). The six groups are as follows: control, diabetes-induced, and 
treatment groups (with silver nitrate (10 $\mathrm{mg} / \mathrm{kg}$ ), M. charantia silver nanoparticles (50 $\mathrm{mg} / \mathrm{kg})$, Metformin (100 $\mathrm{mg} / \mathrm{kg})$, and $M$. charantia aqueous extract $(100 \mathrm{mg} / \mathrm{kg})$ ). The treatment lasted for 12 days after which the animals were sacrificed by cervical dislocation (Elekofehinti et al., 2020). Livers and kidneys of the animals were excised, blotted and put in Eppendorf tubes containing trizol solution.

Gene expression
Total ribonucleic acid (RNA) was isolated after homogenizing the rats' livers and kidneys with TRIzol Reagent (Thermo Fisher Scientific). DNA contaminant was removed following DNAse treatment. The RNA was then quantified and the purity checked using spectrophotometer (A and E Lab UK) at 260 and $280 \mathrm{~nm}$. The RNA was later converted to CDNA using ProtoScript First Strand CDNA Synthesis Kit (NEB). PCR amplicons were gotten using OneTaq $^{\circledR}$ 2X Master Mix (NEB) using the following primer set (Table 1)

Table 1: Primer sets for the gene expression

\begin{tabular}{|c|c|c|}
\hline Gene & Forward primers & Reverse primers \\
\hline KIM-1 & 5 -GGATGAGGATGGGTTTCTTAGG-3' & 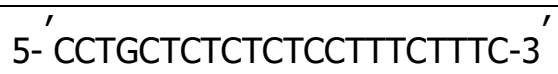 \\
\hline PPARa & 5-' CATCACCCGAGAGTTCCTAAAG-3' & $5^{\prime}$-ATCCAGTTCGAGGGCATTG-3' \\
\hline PPARY & $5^{\prime}$ - CGAGCTGGGAGTAGCCTGA-3' & 5 - GATCACCAGCAGAGGTCCAG-3' \\
\hline $\begin{array}{l}\text { HMG-CoA } \\
\text { reductase }\end{array}$ & $5^{\prime}$ - TGCTGCTTTGGCTGTATGTC- ${ }^{\prime}$ & $5{ }^{\prime}-$ TGAGCGTGAACAAGAACCAG-3' \\
\hline GAPDH & $5^{\prime}$ - AGACAGCCGCATCTTCTTGT-3' & $5^{\prime}$ - CTTGCCGTGGGTAGAGTCAT -3 \\
\hline
\end{tabular}

Polymerase Chain Reaction (PCR)

This has been previously described (Elekofehinti et al., 2020). $5 \mu \mathrm{l}$ of the cDNA template was used to amplify each gene using $2 \mu \mathrm{l}$ of each of the forward and reverse primers with Taq-master mix in a final volume of $15 \mu$ l. The PCR was run as follows: predenaturation for 5 min at $94^{\circ} \mathrm{C}$, annealing for $30 \mathrm{sec}$ at $55^{\circ} \mathrm{C}$ and Extension $72{ }^{\circ} \mathrm{C}$ for $30 \mathrm{sec}$. This was followed by 30 cycles run for $5 \mathrm{~min}$ at $72{ }^{\circ} \mathrm{C}$.

\section{Gel electrophoresis}

The PCR products (amplicons) were electrophoresed in $0.2 \%$ agarose gel using $0.5 \times$ TBE buffer with $3 \mu$ l EZ-vision (VWR Life
Science). The bands were visualized using Blue-light-transilluminator (Blue box U.S.A). The intensities of the bands from agarose gel electrophoresis were quantified densitometrically using Image J software. The results obtained were plotted as bar graphs.

\section{Statistical analysis}

The experimental results were expressed as mean \pm standard error of the mean (SEM) using Graph Pad Prism 8.0.2 and were subjected to one-way analysis of variance followed by post-hoc Dunnett's multiple range tests. Significant levels were tested at $p<0.05$.

\section{Results}

Effect of M. charantia silver nanoparticles on glucose level in streptozotocin- induced diabetic Rats 


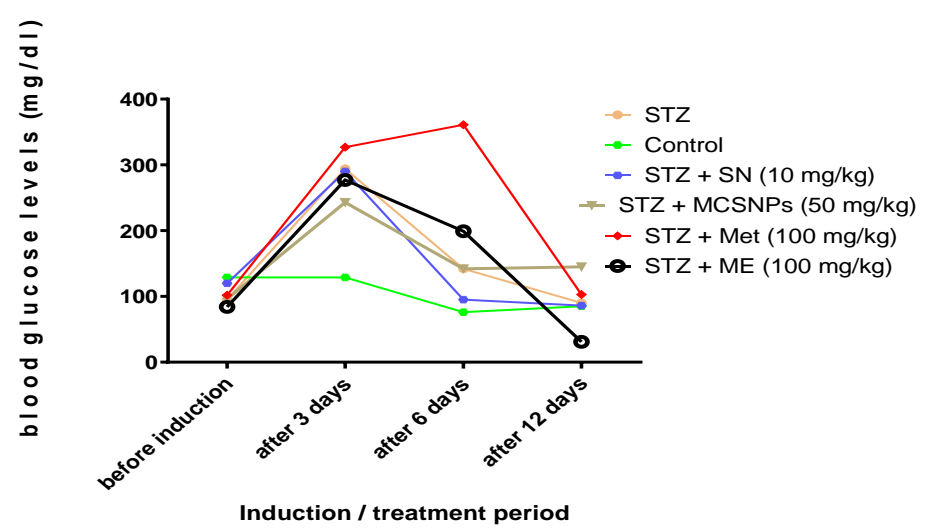

Fig 1: Graph showing the hypoglycemic effect of $M$. charantia nanoparticles on glucose level in streptozotocin-induced diabetic rats. Experiments were conducted in triplicates and all data are presented as Mean \pm SD ( $n=6-8$ animals)

Effect of oral administration of M. charantia silver nanoparticles on 3-hydroxyl, 3-methyl glutaryI_coA (HMG-COA) reductase gene expression in the liver of S-induced diabetic rats

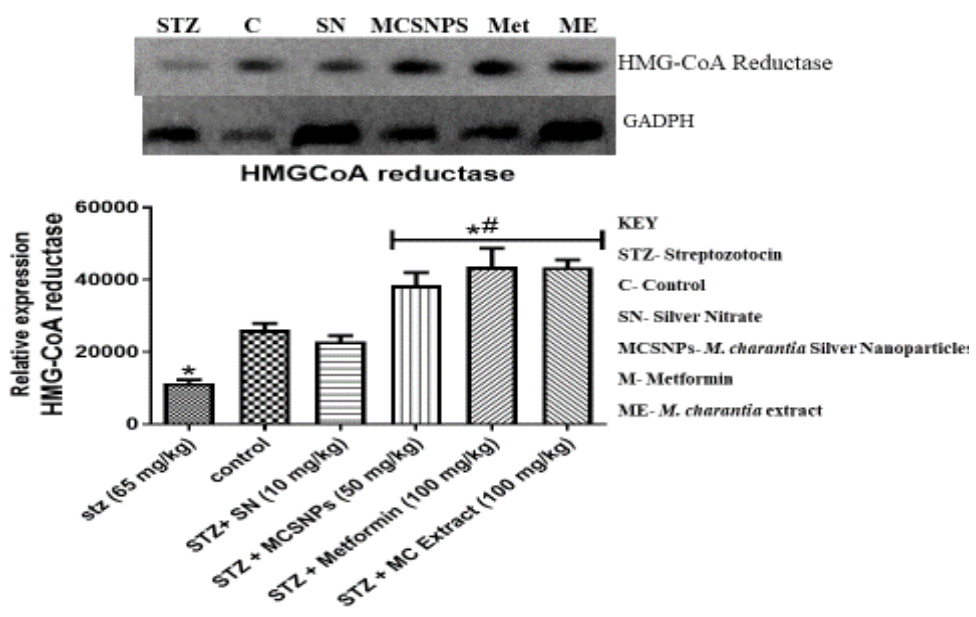

Fig 2: Qualitative-PCR analysis of 3-hydroxyl, 3-methyl glutaryl_coA reductase (HMG-CoA reductase) mRNA expression in STZ-induced diabetic rats. Snapshot representation of RT-PCR agarose gel electrophoresis for HMG-CoA reductase followed by densitometric analysis. *represents significant difference relative to control, \# represents a significant difference relative to diabetic control at $p<0.05$.

Effect of oral administration of M. charantia silver nanoparticles on peroxisome proliferator-activated receptor alpha genes expression in the liver of STZ-induced diabetic rats

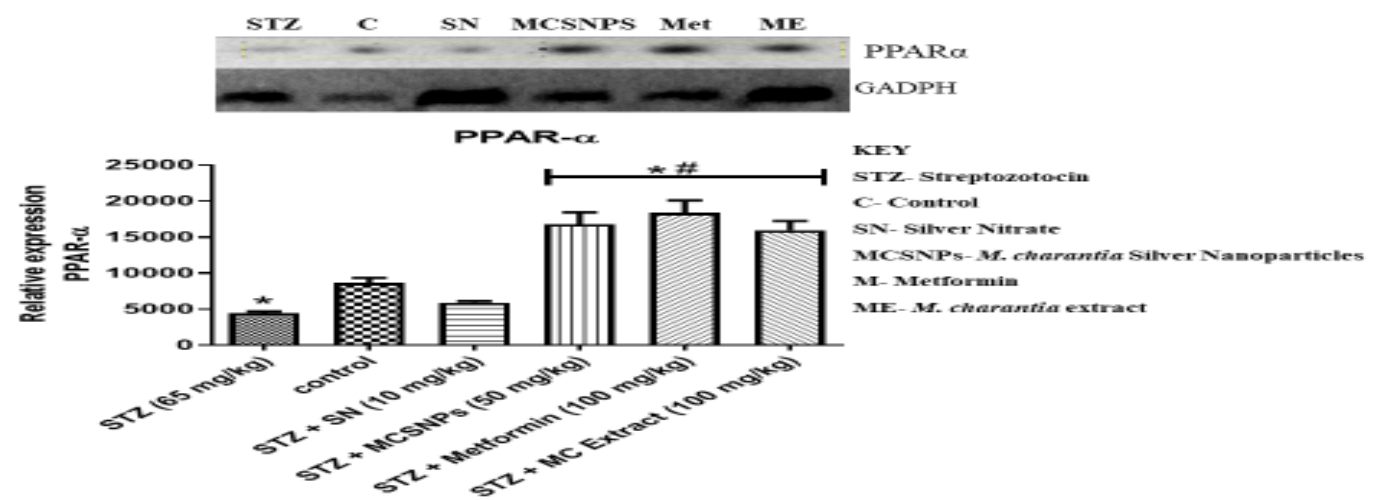


Fig. 3: Qualitative-PCR analysis of peroxisome proliferator-activated receptor alpha (PPARa) gene expression in STZ-induced diabetic rats. Snapshot representation of RT-PCR agarose gel electrophoresis for PPARa followed by densitometric analysis. *represents significant difference relative to control, \# represents a significant difference relative to diabetic control at $p<0.05$.

Effect of oral administration of $M$. charantia silver nanoparticles on peroxisome proliferator-activated receptor gamma (PPARY) genes expression in the liver of STZ-induced diabetic rats

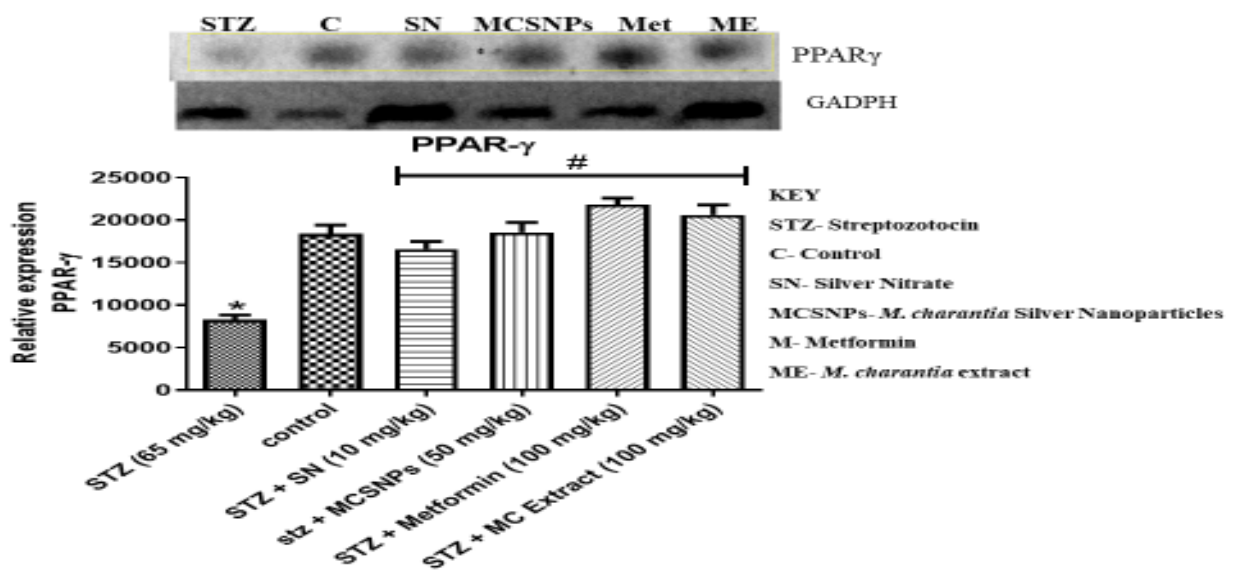

Fig. 4: Qualitative-PCR analysis of peroxisome proliferator-activated receptor alpha and gamma (PPARY) gene expression in STZ-induced diabetic rats. Snapshot representation of RT-PCR agarose gel electrophoresis for PPARy followed by densitometric analysis. *represents significant difference relative to control, \# represents significant difference relative to diabetic control at $p<0.05$

The effect of oral administration of M. charantia silver nanoparticles at different concentrations on kidney injury molecule-1 (Kim-1) gene expression in the kidney of normal and STZ-induced diabetic rats

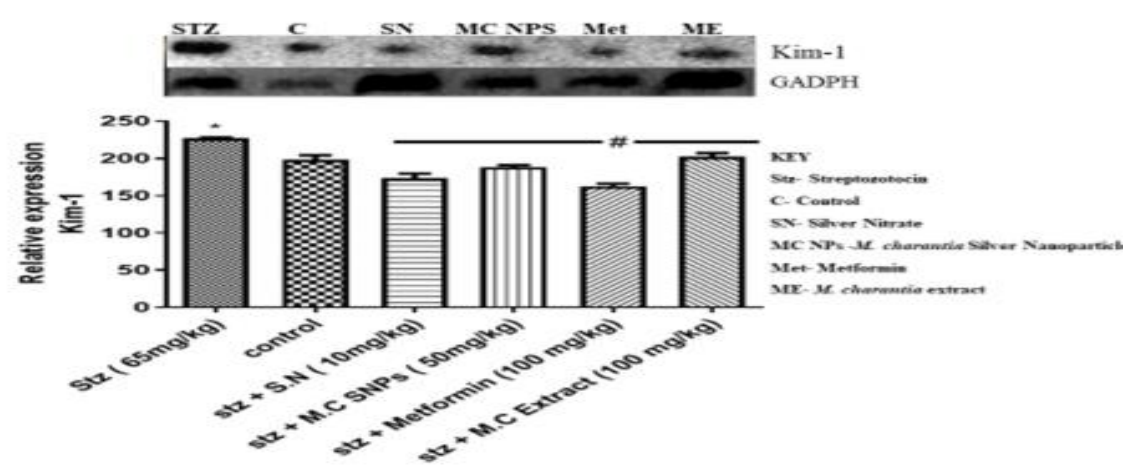

Fig. 5: Qualitative-PCR analysis of Kim-1 gene expression in STZ-induced diabetic rats. Snapshot representation of RT-PCR agarose gel electrophoresis for Kim-1 followed by densitometric analysis. *represents significant difference relative to control, \# represents significant difference relative to diabetic control at $p<0.05$

\section{Discussion}

Diabetes Mellitus (DM) is characterized by hyperglycemia and has also been linked with hypercholesterolemia (a form of hyperlipidemia) which is often the genesis of many cardiovascular diseases such as hypertension, myocardial infarction, stroke and the likes seen in DM complications (Dowarah and Singh, 2020). Another complication is nephropathy; partial or total kidney malfunction which has been reported in DM cases (Mbanya and Sobngwi, 2003). Streptozotocin was used to induce DM in this study since it has been known to cause apoptosis of insulin-producing 
beta-cells of the pancreas resulting in hyperglycemia (Elekofehinti et al., 2020).

The hypolipidemic and antidiabetic effect of Mormodica charantia has been reported (Oishi et al., 2007; Elekofehinti et al., 2018). However, there is dearth of information on its mechanisms and its use with silver nanoparticles as vehicles for treatment. This study investigated the effect of $M$. charantia silver nanoparticles (MCSNPs) on genes associated with lipid metabolism and nephrotoxicity in streptozotocin-induced (STZinduced) diabetic rats. Genes like 3-hydroxyl, 3methyl glutaryl_coA reductase (HMG-CoA reductase), peroxisome proliferator-activated receptor alpha and gamma (PPARa and PPARY) were investigated in the liver of MCSNPs treated rats since liver is central in lipid metabolism (Staels and Fruchart 2005). Liver stores excess fatty acids as triacyl glycerides (TAG) and it is also responsible for production of bile acids. Kidney injury molecule 1 (KIM-1), a sensitive marker gene associated with nephrotoxicity was also examined in the kidney. The blood glucose of the rats was significantly $(p<0.05)$ elevated after 72 hours (3 days) of induction with streptozotocin $(65 \mathrm{mg} / \mathrm{kg})$ as seen in Fig 1 above. This is because streptozotocin (STZ) has the ability to destroy the insulin producing cells in the pancreas resulting in elevated blood sugar since insulin together with the liver regulates glucose and fatty acid metabolism (Al-Qattan et al., 2008). $M$. charantia nanoparticles (MCSNPs) and $M$. charantia plant extract (MC.E) significantly $(p<0.05)$ lowered blood glucose of diabetic rats after 12 days of treatment. This could imply that the plant resuscitated beta cells of the pancreas enabling more insulin to be produced thus ensuring glucose homeostasis. Stimulation of insulin secretion of $M$. charantia has also been reported (Keller et al., 2011).

3-hydroxyl, 3-methyl glutaryl coA reductase (HMG-CoA reductase) is a rate-controlling enzyme of the mevalonate pathway that produces cholesterol (Cherng et al., 2008). The expression of liver HMG-CoA reductase was significantly $(p<0.05)$ down-regulated in STZinduced group relative to control as seen in Fig 2. This could result to hypercholesterolemia and hyperglycaemia seen since cholesterol synthesis pathway is not regulated. Continuous synthesis of cholesterol in the long term may result in its accumulation on receptors leading to insulin resistance. Decrease in the activity of HMG-CoA reductase has also been reported in diabetes cases (Xiujuan et al., 2015). M. charantia silver nanoparticles significantly $(p<0.05)$ upregulate the expression of HMGCoA reductase relative to diabetic group. This could help in regulation of cholesterol synthesis via the feedback mechanism caused by accumulation of mevalonate in the cholesterol biosynthesis pathway. Inhibition of sterol production by accumulation of mevalonate as also been reported (DeBose-Boyd, 2008).

Peroxisome proliferator-activated receptors (PPARs) is a superfamily of transcription factors which include isoforms such as PPAR-alpha and PPAR-gamma (Florian et al., 2006). PPAR-alpha modifies insulin resistance and ensures glucose homeostasis in the liver while PPARgamma regulates the genes involved in lipid metabolism (Florian et al., 2006). PPARalpha and PPAR-gamma were significantly $(p<0.05)$ down-regulated in the STZ-induced group relative to the control as seen in Fig 3 and 4. This could be the reason for the hyperglycemia and hypercholesterolemia seen in the STZ-induced group since these genes ensure glucose and fatty acid homeostasis. $M$. charantia nanoparticles (MCSNPs) and $M$. charantia aqueous extract (MC.E) significantly $(p<0.05)$ up-regulated the expression of PPARalpha and PPAR-gamma compared to STZ group. This may be due to these genes ensuring fatty acid storage and enhancing insulin secretion and function as supported by the work of Chigurupati et al. (2015). There is no significant difference between both MCSNPs (50 $\mathrm{mg} / \mathrm{kg}$ ) and M. charantia extracts (100 $\mathrm{mg} / \mathrm{kg}$ ) for both genes, indicating that silver nanoparticle could ensure bioavailability at a lower dosage of $M$. charantia.

Kidney injury molecule-1 (KIM-1) proteins are secreted by the kidney into the urine and have been found to be sensitive markers of acute kidney injury (Van Timmeren et al., 2007). Kidney injury molecule-1 (KIM-1) gene expression (Fig. 5) was up-regulated in STZinduced test rats when compared to control rats. This showed that hyperglycemia could be capable of inducing nephrotoxicity. KIM-1 gene expression was downregulated in MCSNPs and $M$. charantia extracts relative to the diabetic control rats. This could mean the plant has ameliorative effect on diabetes -induced kidney malfunction .

\section{Conclusion}

This study revealed that $M$. charantia silver nanoparticles (MCSNPs) and $M$. charantia extracts administered to streptozotocin-induced diabetic rats improves or ameliorates diabetes 
conditions by up-regulating the expression of HMG-CoA reductase, PPAR-alpha and PPARgamma coupled with down-regulating KIM-1, a gene associated with nephrotoxicity. However, there is no significant difference in the modulation of genes in both MCSNPs $50 \mathrm{mg} / \mathrm{kg}$ and M. charantia extracts $100 \mathrm{mg} / \mathrm{kg}$ groups. This work revealed that silver nanoparticle technology ensures bioavailability of $M$. charantia at less dosage $(50 \mathrm{mg} / \mathrm{kg})$. The study further indicated that coupling drugs with silver nanoparticles as seen in administration of M. charantia silver nanoparticles could be an improved, dosage-effective and viable alternative in the management of diabetes mellitus and its complications.

\section{References}

Abdelwahed, W., Degobert, G., Stainmesse, S. and Fessi, H. (2006). Freeze-drying of nanoparticles: formulation, process and storage considerations. Adv Drug Deliv Rev.; 58(15):1688-1713.

Akbarzadeh, A., Norouzian, D., Mehrabi, M.R., Jamshidi, S.H., Farhangi, A., Allah, V.A., Mofidian, S.M.A. and Lame, R.B. (2007). Induction of diabetes by streptozotocin in rats. Indian Journal of Clinical Biochemistry, 22(2): 60-64.

Ahn, K. (2017). The worldwide trend of using botanical drugs and strategies for developing global drugs. BMB Reports. 50 (3): 111-116.

Al-Qattan, K., Thomson, M. and Ali, M. (2008). Garlic (Allium sativum) and ginger (Zingiber officinale) attenuate structural nephropathy progression in streptozotocin-induced diabetic rats; Europeran Journal Clin Nut Metab; 3: 6271.

Bharathi, V., Jannathul, F., Noorzaid, M. and Resni, M. (2017): Green synthesis of Mangifera indica silver nanoparticles and its analysis using Fourier transform infrared and scanning electron microscopy; National Journal of Physiology, Pharmacy and Pharmacology, 7 (12): 1-4.

Bloomgarden Z.T. (2003). Cardiovascular disease and diabetes; Diabetes Care 26(1), 230-237.

Cherng, S., Young, J. and Ma, H. (2008). HMG$\mathrm{CoA}$ reductase (3-hydroxy-3-methyl-glutaryl-
CoAreductase) (HMGR); The Journal of American Science; 4(3): 61-64.

Chigurupati, S., Dhanaraj, S.A. and Balakumar, P. (2015). A step ahead of PPARy full agonists to PPARy partial agonists: Therapeutic perspectives in the management of diabetic insulin resistance; European Journal of Pharmacology, 755: 50-57.

DeBose-Boyd R. A. (2008): Feedback regulation of cholesterol synthesis: sterol-accelerated ubiquitination and degradation of HMG CoA reductase. Cell research, 18(6): 609-621.

Dowarah, J. and Singh, V.P. (2020). Antidiabetic drugs recent approaches and advancements. Bioorg Med Chem.28(5): 115263.

Elekofehinti, O.O., Ariyo, E.O., Akinjiyan, M.O., Olayeriju, O.S., Lawal, A.O., Adanlowo, I.G. and Rocha, J.B.T. (2018). Potential use of bitter melon (Momordica charantia) derived compounds as antidiabetics: in silico and in vivo studies; Pathophysiology, 945: 1-7.

Elekofehinti, O.O., Omotuyi, I.O., Kamdem, J.P., Alves, V.G., Ejelonu, O.C., Adanlawo, I.G. and Rocha, J.B.T. (2014). Saponin as regulator of biofuel: Implication for ethnobotanical management of diabetes. J Physiol Biochem; 70(2): 555-567.

Elekofehinti, O.O., Onunkun, A.T. and Olaleye, M.T. (2020). Cymbopogon citratus (DC) Stapf mitigates ER-stress induced by streptozotocin in rats via down-regulation of GRP78 and Upregulation of NRF2 signaling. Journal of Ethnopharmacology 262: 113130.

Florian, B., Yasunori, T. and Evren, C. (2006). Obesity, Peroxisome Proliferator-Activated Receptor, and Atherosclerosis in Type 2 Diabetes; Arteriosclerosis, Thrombosis, and Vascular Biology, 26: 28-40.

Hui, H., Tang, G. and Go, V.L. (2009). Hypoglycemic herbs and their action mechanisms. Chin Medn; 4:11.

Keller, A.C., Ma, J., Kavalier, A., He, K., Brillantes, A.M. and Kennelly, E.J. (2011). Saponins from the traditional medicinal plant Momordica charantia stimulate insulin secretion in vitro; Phytomedicine; 19:32-37. 
Kitabchi A.E., Umpierrez G.E., Miles J.M. and Fisher J.N. (2009). Hyperglycemic crises in adult patients with diabetes; Diabetes Care; 32(7): 1335-43.

Mbanya J.C. and Sobngwi E. (2003). Diabetes microvascular and macrovascular disease in Africa; European Journal of Cardiovascular Risk, 10(2): 97-102.

Oishi, Y., Sakamoto, T., Udagawa, H., Taniguchi, H., Kobayashi-Hattori, K., Ozawa, Y. and Takita, T., (2007). Inhibition of increases in blood glucose and serum neutral fat by Momordica charantia saponin fraction. Biosci. Biotechnol. Biochem. 71,735-740.

Omonkhua, A.A., Onoagbe, I.O., Fajimeye, I.A., Adekola, M.B., Imoru, Z.A. (2012). Long term antidiabetic and antihyperlipidaemic effects of aqueous stem bark extracts of Irvingia gabonesis in streptozotocin-induced diabetic rats. Asian Pacific J Tropi. Biomed; 2:1-6.

Patel., S., Patel, T., Parmar, K., Bhatt, Y., Patel, Y. and Patel, N.M.D. (2010). Isolation, characterization and antimicrobial activity of charantin from Momordica charantia linn. Fruit. Int J Drug Deve Res. 2(3): 629-634.

Shameli, K., Ahmad, M.B., Yunus, W.M.Z.W., Ibrahim, N.A., Gharayebi, Y. and Sedaghat, S. (2010). Synthesis of silver/montmorillonite nanocomposites using $\mathrm{Y}$-irradiation. Int. $\mathrm{J}$. Nanomed.; 5:1067-1077
Shaw, J.E., Sicree, R.A. and Zimmet, P.Z. (2010). Global estimates of the prevalence of diabetes for 2010 and 2030; Diabetes Research and Clinical Practice; 87: 4-14.

Staels, B. and Fruchart, J.C. (2005). Therapeutic roles of peroxisome proliferatoractivated receptor agonists. Diabetes.;54(8):2460-2470.

Van Timmeren, M.M., Vaidya, V.S., Van Ree, R.M. (2007). High urinary excretion of kidney injury molecule-1 is an independent predictor of graft loss in renal transplant recipients. Transplantation, 84:1625-1630.

World Health Organization (2006). Definition and diagnosis of diabetes mellitus and intermediate hyperglycemia: report of a WHO IDF consultation.

Xiujuan, Z., Yongfeng. S., Mei, F., Xinli, Z., Ling, Gao., Chunxiao, Y., Xiuyun, J., and Jiajun, Z. (2015). Thyroid-Stimulating Hormone Decreases HMG-CoA Reductase Phosphorylation via AMP-Activated Protein Kinase in the liver; Journal of Lipid metabolism; 56(5): 1-36.

Zhang, X. F., Liu, Z. G., Shen, W. and Gurunathan, S. (2016). Silver Nanoparticles: Synthesis, Characterization, Properties, Applications, and Therapeutic Approaches. International journal of molecular sciences, 17(9): 1534. 